\title{
Les politiques éducatives aux États-Unis
}

Entre privatisation et investissement public

Education policies in the United States. Between privatization and public investment

Las políticas educativas en Estados Unidos. Entre privatización e inversión pública

\section{Frank Adamson}

Traducteur : Sylvaine Herold

\section{CpenEdition} Journals

Édition électronique

URL : https://journals.openedition.org/ries/6071

DOI : 10.4000/ries.6071

ISSN : 2261-4265

Éditeur

France Education international

Édition imprimée

Date de publication : 1 décembre 2017

Pagination : 91-102

ISBN : 978-2-85420-616-6

ISSN : 1254-4590

Référence électronique

Frank Adamson, "Les politiques éducatives aux États-Unis », Revue internationale d'éducation de Sèvres [En ligne], 76 | décembre 2017, mis en ligne le 01 décembre 2019, consulté le 24 juin 2021. URL http://journals.openedition.org/ries/6071 ; DOI : https://doi.org/10.4000/ries.6071 


\title{
Les politiques éducatives aux États-Unis*
}

\section{Entre privatisation et investissement public}

\author{
Frank Adamson \\ Stanford University
}

Le système éducatif des États-Unis est actuellement à un tournant décisif. Les décideurs politiques ont longtemps débattu de la meilleure façon d'améliorer la qualité de l'éducation et de combler l'importance des écarts de résultats entre élèves. Si les disparités liées à l'origine ethnique et sociale des élèves ne sont pas contestées, les pistes de solution demeurent fortement controversées. Deux approches sont principalement mises en œuvre: des systèmes fondés sur le marché, qui privatisent la gestion des établissements d'enseignement et des approches en termes de renforcement des capacités, intensifiant au contraire les investissements publics.

Les partisans des approches fondées sur le marché défendent la liberté de choix en matière éducative. Pour certains, l'idée principale est que la concurrence forcera les établissements d'enseignement à proposer de bons produits ou à "fermer boutique », tout en offrant aux familles la possibilité de choisir entre différents établissements via des dispositifs tels que les chèques éducation (school vouchers) ou les charters schools ${ }^{1}$. Pour d'autres, l'objectif est de créer des opportunités d'innovation et de proposer des options variées aux élèves, afin qu'ils puissent parvenir à un ajustement optimal de leurs intérêts. La Nouvelle-Orléans, en Louisiane, est un exemple type de cette option politique : s'y déroule actuellement la plus grande initiative en cours en matière de privatisation de l'éducation aux États-Unis, via l'expansion des charter schools à l'ensemble du système.

D'autres réformes mettent au contraire l'accent sur l'investissement public en faveur de la formation professionnelle et de l'égalisation du financement et des possibilités curriculaires comme stratégie pour combler les écarts de réussite (Darling-Hammond et al., 2005 ; Elmore et Burney, 1997 ; Jackson et al., 2014). L'État du Massachusetts constitue un bon exemple de ce type de stratégie.

\footnotetext{
* Article traduit par Sylvaine Herold.

1. Vouchers : « chèques éducation donnés par le gouvernement fédéral aux parents désirant retirer leur enfant d'une école publique jugée défaillante pour l'inscrire dans une école privée "; les charter schools « sont des écoles à financement public mais à gestion privée sous contrat (charter) : en échange d'une plus grande autonomie de fonctionnement, la charter school s'engage à atteindre certains objectifs qui, s'ils ne sont pas atteints au bout de trois ou cinq ans, peuvent entraîner la révocation du contrat et la fermeture de l'école. " D'après L. Emile-Besse (2014), "Le système éducatif américain ", Revue internationale d'éducation de Sèvres n 35 , [en ligne : http://ries.revues. org/1760 ; DOI : 10.4000/ries.1760]. (NdIR)
} 
Cet article examine l'évolution de ces deux perspectives en termes de politique, de pratique et de résultats, à travers les cas de la Nouvelle-Orléans et du Massachusetts. Ce travail de recherche n'établit aucun lien de causalité entre les orientations politiques et l'état de ces systèmes éducatifs mais illustre la manière dont les contextes politiques à long terme façonnent les systèmes éducatifs. Ces systèmes représentent en outre des niveaux différents de différenciation, le système éducatif de la Nouvelle-Orléans devenant à l'heure actuelle un marché extrêmement fragmenté d'établissements d'enseignement gérés en grande partie par des organismes privés, à mesure que le rôle de l'État est réduit à une simple fonction d'accréditation, tandis que l'État du Massachusetts conserve un rôle important. Par ailleurs, les établissements d'enseignement de la Nouvelle-Orléans sont contraints de participer à une vision étroite de la scolarisation, qui se concentre sur la préparation aux examens en mathématiques et en anglais, au détriment d'une conception plus vaste de l'éducation ; le Massachusetts est, quant à lui, parvenu à préserver un éventail plus vaste d'offres curriculaires, intégrées à ses structures d'État.

\section{DES PARADIGMES ÉCONOMIQUES CONCURRENTS}

Les États-Unis ont toujours été marqués par des élans contraires - une demande d'équité entrant en tension avec des forces en faveur de la concentration des richesses et du pouvoir. Dans les secteurs sociaux et éducatifs, cette demande d'équité est survenue par cycles au cours de l'histoire américaine, succédant la plupart du temps à des périodes où les inégalités étaient devenues extrêmes. Dans l'histoire récente, les années 1960 et 1970 ont ainsi été le théâtre des protestations en faveur des droits civiques, consécutives à la prise de conscience des inégalités, qui ont finalement conduit aux réformes en faveur de la déségrégation et du financement de l'école, afin d'accroître l'équité en éducation. Cette époque a également été celle de la presque disparition de l'écart entre les minorités ethniques et les blancs s'inscrivant dans l'enseignement supérieur, grâce à des aides financières accrues conjuguées à des mesures de discrimination positive.

Mais depuis les années 1980, l'approche conservatrice a dominé le paysage politique, entraînant l'augmentation massive des inégalités à la fois en termes de revenus et de résultats éducatifs (Adamson, 2010). Cette hausse des inégalités a coïncidé avec l'adoption généralisée d'une approche néolibérale dans les sphères politiques et économiques - et en résulte d'une certaine façon. Ces changements ont eu lieu dans le contexte des nouvelles angoisses économiques qui ont émergé dans les années 1970, liées notamment au phénomène nouveau de la "stagflation", qui combine des taux d'intérêt et un chômage élevés avec un taux de croissance faible. Le malaise lié à la stagflation a laissé le champ libre à l'influence du principal partisan de l'économie de "libre marché », Milton Friedman. Ce dernier soutenait notamment que le rôle premier de l'État devait 
être de garantir le bon fonctionnement du marché et que « les politiques monétaires devaient prévaloir sur les politiques fiscales (fiscalité et redistribution) conçues par le "Grand gouvernement"2»(Steger et Roy, 2010). Friedman était également un ardent défenseur du système de chèques éducation, au nom de la liberté de choix éducatif et de la libre concurrence - une idée qu'il a notamment exportée au Chili dans les années 1970 et proposée aux États-Unis dans les années 1980.

\section{NÉOLIBÉRALISME ET ÉDUCATION}

Le principe néolibéral de primauté des intérêts privés sur la gouvernance publique et un certain accommodement avec l'inégalité des chances se sont traduits dans la sphère éducative de plusieurs façons :

- l'appui au financement public des choix privés, à travers les chèques éducation et, par la suite, les charter schools, qui sont parfois autorisées à sélectionner les élèves ;

- la résistance à la péréquation des financements entre établissements publics, qui permettrait une plus grande équité dans les opportunités éducatives ;

- l'utilisation généralisée d'un système de reddition de comptes basé sur les tests, comme moyen d'évaluer les établissements les uns par rapport aux autres et de décider lesquels doivent être maintenus ou fermés ;

- la création d'un marché non réglementé d'enseignants, au lieu d'un système de pré-requis professionnels (ce qui permet de réduire à la fois les salaires et les standards, en particulier dans les communautés les plus démunies).

Cet article s'intéresse spécifiquement à l'exemple des charter schools. À l'origine, l'idée de "mettre sous contrat » des établissements d'enseignement a reçu le soutien des dirigeants progressistes. Ainsi, le dirigeant du syndicat enseignant Al Shanker pensait que les charter schools pourraient favoriser l'innovation dans le secteur public. Suite à la première loi autorisant la création de charter schools dans l'État du Minnesota en 1991, 42 États ainsi que le district de Columbia avaient adopté, pour l'année scolaire 2012-2013, des législations en faveur des charter schools. De 2000 à 2013, le nombre d'élèves inscrits dans des charter schools publiques est ainsi passé de 0,3 million à 2,3 millions, pour représenter 4,6 \% de l'ensemble des élèves en 2013 (NCES, 2015). En 2014, les inscriptions dans les charter schools étaient constituées à $58 \%$ d'élèves afro-américains et latinos, tandis que ces populations ne représentaient que $41 \%$ des effectifs dans les établissements publics (ibid.). Avec le temps, le mouvement des charter schools a évolué : ce ne sont désormais plus des établissements isolés créés par des groupes aux idées éducatives particulières, mais des chaînes d'établissements gérées par des "organisations de gestion des charter schools" (charter management organizations), dont certaines opèrent en tant qu'entreprises à but lucratif.

2. Big government en anglais. (NdT) 


\section{LA POLITIQUe de PRIVATISATION DE LA NOUVELLE-ORLÉANS}

La Nouvelle-Orléans est la première ville à avoir complètement privatisé le fonctionnement des établissements scolaires au niveau du district. Elle comptait près de 400000 habitants en 2015 et plus de 40000 élèves. En 2014, plus de 85 \% des élèves étaient afro-américains et $84 \%$ d'entre eux étaient économiquement défavorisés, un taux de plus de $20 \%$ supérieur à la moyenne de l'État. Le système scolaire de la Nouvelle-Orléans et de la Louisiane a en outre une longue histoire d'injustice, à laquelle le système des charter schools était censé remédier.

En 2003 - avant l'ouragan Katrina -, la Louisiane a mis en œuvre un dispositif de "district scolaire de rattrapage "(Recovery School District, RSD), dans l'objectif de prendre en charge les établissements scolaires désignés comme défaillants selon la loi fédérale No Child Left Behind (NCLB). Au cours des deux premières années du RSD, l'État a proposé la mise sous contrat de certains établissements, comme une option possible. Puis, en 2005, l'ouragan Katrina a dévasté la ville, emportant des vies, détruisant des biens et déplaçant un grand nombre de personnes. Après Katrina, le paysage éducatif avait radicalement changé et la Nouvelle-Orléans est devenue la première ville des États-Unis à supprimer les établissements publics de district.

L'exemple de la Nouvelle-Orléans est particulièrement important, car les tenants de la privatisation l'ont présenté comme une expérience éducative ambitieuse pouvant servir de modèle aux autres écoles de district du pays. Dans le monde de la recherche scientifique toutefois, les expériences se fondent habituellement sur des projets pilotes réussis, des groupes témoins et des possibilités d'abandon. Rien de tout cela n'a existé dans le cas présent. Les enfants ont au contraire été les sujets captifs d'une décision visant à mettre un terme à leur système d'enseignement public. Et, outre le fait qu'aucun processus organisé d'évaluation n'a jusqu'ici accompagné ce projet de réforme radical, l'État de Louisiane a également refusé de divulguer des données publiques sur les élèves et leurs résultats, n'acceptant de les fournir qu'aux deux institutions qui collaborent avec les réformateurs ${ }^{3}$.

Bien qu'il soit très difficile de déterminer l'impact de la mise en œuvre de ce système de charter schools, étant donné le changement démographique radical après Katrina et l'absence d'accès à des données au niveau des élèves qui permettraient des analyses comparatives entre groupes, il apparaît néanmoins clairement que les résultats scolaires continuent d'être faibles dans le district de la Nouvelle-Orléans. Seuls $12 \%$ des élèves du RSD ont obtenu des résultats du

\footnotetext{
3. Après cinq années durant lesquelles le département de l'éducation de Louisiane a rejeté les demandes répétées de données formulées par des chercheurs de la Nouvelle-Orléans préoccupés par les effets des réformes, une organisation dénommée "Recherche sur les réformes » [Research on Reforms] a intenté un procès contre l'État. Après plusieurs années de procès, la justice a finalement rendu une décision en sa faveur, en 2015. Même après cette décision favorable, l'organisation n'a pas été en mesure d'accéder à des données. Voir : [https://goo.gl/8q4ijk]
} 
niveau «maîtrise » ou plus lors des examens étatiques de 2014, soit la moitié du taux de l'ensemble de l'État (24\%). En revanche, dans le district des écoles paroissiales de la Nouvelle-Orléans (Orleans Parish Schools) - un district désormais distinct de la Nouvelle-Orléans, où opèrent des écoles publiques et des charter schools hautement sélectives -, cette part était bien supérieure (42\%).

Dans le même temps, des recherches de terrain, dont certaines menées par nous, indiquent que, pour un grand nombre de familles de la NouvelleOrléans, la mise en œuvre d'un modèle concurrentiel fondé sur le marché a créé un système de gagnants et de perdants. Cela a également accru la stratification entre établissements et exclut des options les plus désirables les élèves considérés comme «à risque ». Ce processus a en outre exacerbé des inégalités de résultats déjà importantes. L'un des parents que nous avons interrogés à la NouvelleOrléans nous a fait part de sa vision, désastreuse, de la situation :

On va avoir ici [à la Nouvelle-Orléans] une classe [sociale] qui aura été éduquée et une classe qui ne l'aura pas été. C'est ça la réalité qu'on observe aujourd'hui. Ce que je veux dire, c'est que dans une génération on va avoir ici une sous-classe comme il n'en existe nulle part ailleurs dans le pays, parce qu'une partie de la population n'aura pas reçu d'éducation, et si la population n'est pas éduquée, alors on ne peut pas avoir de démocratie, et c'est ça le problème.

De l'avis de ce parent, également relayé par d'autres, la privatisation de l'éducation a réduit les opportunités éducatives pour un grand nombre d'enfants, avec de graves conséquences à long terme pour l'avenir politique et social de la Nouvelle-Orléans.

\section{La conversion des établissements publics de la Nouvelle-Orléans en charter schools}

Milton Friedman ne préconisait pas un changement progressif, il pensait plutôt qu'un "choc » économique était le plus à même de changer le système. À la Nouvelle-Orléans, ceux qui cherchaient le moyen de privatiser le système éducatif ont considéré que la crise engendrée par l'ouragan Katrina était le "choc» opportun. Peu de temps après le passage de l'ouragan, le Conseil d'éducation de l'État (State Board of education) a adopté la Loi 35, qui a considérablement étendu la définition de ce qui était entendu par établissement scolaire défaillant, tout en fournissant au RSD le mandat légal pour prendre en charge ou externaliser le fonctionnement de ces établissements. Le Conseil d'éducation de la Louisiane a ainsi utilisé Katrina et la Loi 35 pour transférer la supervision du système éducatif des mains des habitants et des élus locaux de la Nouvelle-Orléans vers celles des fonctionnaires de l'État et, dans bien des cas, vers les conseils d'administration non élus des organisations de gestion des charter schools. L'État de Louisiane, agissant par le biais du RSD, a ensuite sollicité 
des organismes de gestion extérieurs à l'État pour gérer les établissements scolaires de la Nouvelle-Orléans, ce qui constitue aujourd'hui un grief majeur pour les habitants rapatriés de la ville. Le chef d'établissement d'une charter school que nous avons interrogé considère que le district a été "volé " au cours de ce processus.

Pendant la période de transition pour la mise en ouvre du système de charter schools, l'État de Louisiane a également supprimé plus de 7000 postes d'enseignants titulaires. Ces derniers n'ont pas bénéficié de la procédure régulière et la plupart d'entre eux n'ont pas eu la possibilité de postuler de nouveau à leur poste, alors même que leur contrat stipulait qu'une liste d'enseignants serait créée et utilisée en cas de réembauche. Au lieu de quoi, un grand nombre de charter schools ont traité avec l'organisation Teach for America [Des enseignants pour l'Amérique], faisant venir de jeunes enseignants inexpérimentés sous contrat de deux ans et des administrateurs venus d'autres horizons géographiques afin d'instituer, bien souvent, un modèle de tolérance zéro dans les charter schools.

Par cette stratégie, l'État de Louisiane a retiré aux élus la responsabilité de l'éducation publique pour la confier aux conseils d'administration d'organismes de gestion privés et de bailleurs de fonds extérieurs à l'État, privant ainsi de leurs droits les élèves, les parents et les enseignants.

\section{Un choix inversé : comment les établissements sélectionnent leurs élèves}

L'une des hypothèses de base des modèles fondés sur le marché est que les consommateurs sont libres de choisir les produits auprès de fournisseurs en concurrence entre eux. En théorie, opposer les performances des établissements fournirait aux familles une mesure de comparaison, ce qui devrait en retour inciter les établissements à s'améliorer s'ils ne veulent pas courir le risque de perdre des effectifs et, finalement, de fermer. Pour les défenseurs du marché, ce système permettrait d'éliminer efficacement les établissements les moins performants. Mais cette situation idéalisée suppose que tous les consommateurs soient des clients également désirables, pour lesquels les fournisseurs sont prêts à entrer en concurrence, et elle présuppose également une information parfaite des parents, la capacité des élèves à changer d'établissement à tout moment et celle des établissements à adapter leurs approches, malgré les disparités de financement mentionnées précédemment et les besoins variés des élèves.

En outre, comme les systèmes d'évaluation fédéraux et étatiques sont centrés sur les résultats des élèves aux examens - le premier critère pris en compte dans les classements -, la survie et l'avantage compétitif d'un établissement sont liés à ses résultats. Cela crée donc une incitation à attirer et à ne retenir que les meilleurs élèves, et à exclure ceux qui feraient baisser la moyenne - une approche 
dénommée "écrémage ». En conséquence, en lieu et place d'un marché de concurrence parfaite entre établissements favorisant le choix parental, ces dix dernières années à la Nouvelle Orléans ont plutôt vu s’accroître la stratification entre établissements, qui désavantage encore plus les élèves en difficulté.

Dans ce système qui autorise "l'écrémage ", on peut distinguer trois niveaux d'établissements. Les établissements du « niveau 1 » utilisent différents critères pour sélectionner à l'entrée, par exemple des tests d'admission et des préférences en termes de fratrie et de quartier. Ces établissements performants reçoivent des milliers de candidatures chaque année, dont ils ne sélectionnent qu'une faible partie. Ils sont fréquentés par l'écrasante majorité des élèves blancs inscrits dans les écoles publiques de la Nouvelle-Orléans (environ 90 \%). Les établissements secondaires supérieurs de ce niveau sont fréquentés par moins d'un tiers des élèves afro-américains ou bénéficiant de repas à prix réduits ou gratuits, qui constituent la grande majorité des élèves dans les autres établissements. Dans ces établissements très performants, les élèves identifiés comme ayant des «besoins particuliers » sont généralement les élèves dits "doués » ou « talentueux », une situation très différente de celle des établissements des autres niveaux, où l'on retrouve plutôt sous cette appellation les élèves « émotionnellement perturbés " ou «autistes ".

Le second niveau d'établissements est constitué des charter schools du RSD. Ces établissements sont engagés dans différentes stratégies d'écrémage afin de limiter le nombre d'élèves situés dans la partie basse de la courbe de performance. Selon différents rapports et des entretiens menés auprès de parents et d'élèves ayant postulé à ces établissements, ces stratégies comprennent a minima les éléments suivants : 1) le refus d'inscrire les élèves les moins performants et ceux ayant des antécédents disciplinaires ou des besoins éducatifs particuliers ; 2) le renvoi de certains élèves en octobre après avoir reçu la subvention annuelle de l'État ; 3) le recours à des arrestations d'élèves dans l'enceinte de l'école, ce qui les exclut de fait sans que l'établissement n'ait à les expulser.

Le troisième niveau, le plus bas, est celui des écoles alternatives. Ces établissements sont les établissements de la dernière chance pour les élèves, avant qu'ils soient complètement exclus du système scolaire; ils sont également un point de réadmission pour les jeunes issus du système correctionnel. À la Nouvelle-Orléans, une société gérant des établissements correctionnels dans d'autres États est en charge de la gestion d'une de ces charter schools alternatives. Cette confusion des genres entre, d'une part, un système éducatif stratifié et, d'autre part, le système correctionnel d'État montre à quel point les charter schools de la Nouvelle-Orléans font partie intégrante d'un circuit " de l'école à la prison », pour les élèves les plus désavantagés.

À la Nouvelle-Orléans, qui plus est, les résultats des élèves décroissent selon ces niveaux, ce qui crée un système stratifié dans lequel les élèves qui sont amenés à changer d'établissement le font principalement à sens unique, vers les établissements les moins performants. Les élèves n’ont pas la possibilité de 
décider de passer un test d'admission en milieu d'année, afin d'entrer dans l'un des établissements publics les plus performants, à majorité blanche. Mais les élèves peuvent tout à fait être renvoyés d'une charter school peu performante pour être dirigés vers une école alternative du troisième niveau, le plus bas du RSD. Dans ce système stratifié, lorsque les établissements disponibles pour les élèves en difficulté font preuve d'un faible niveau de performance, ils sont fermés et leurs élèves envoyés dans d'autres établissements. Mais de plus en plus de rapports suggèrent que ces élèves aboutissent en fait dans des établissements tout aussi défaillants (Williams, 2013). Notons tout de même que cette stratification accrue présente vraisemblablement des avantages pour les élèves ayant la chance de fréquenter l'une des charter schools privilégiées du premier niveau.

En substance, l'exemple de la Nouvelle-Orléans montre qu'adopter des politiques de privatisation de l'éducation en mettant en place des charter schools a certes intensifié la concurrence entre établissements, mais a également : 1) accru la stratification entre établissements et la ségrégation, 2) réduit les options disponibles pour les élèves aux besoins les plus importants et créé un secteur indésirable - couramment désigné sous le terme d' "établissements poubelles » - pour ces jeunes, et 3) encouragé l'exclusion pure et simple de l'école des élèves en difficulté. Pour les élèves non rentables, le marché semble de fait ne pas offrir de choix.

\section{UNE STRATÉGIE D'INVESTISSEMENT PUBLIC DANS LE MASSACHUSETTS}

Il est intéressant de noter que les États obtenant les meilleurs résultats aux États-Unis - Connecticut, Massachusetts, New Hampshire, New Jersey et Vermont - figurent également parmi ceux ayant eu le moins recours à des réformes fondées sur le marché.

Parmi ces États, le Massachusetts est un exemple particulièrement instructif. Cet État comptait environ 6,5 millions d'habitants en 2016 et un peu plus de 950000 élèves, dont près d'un tiers économiquement défavorisé. Certes, la situation diffère de celle de la Nouvelle-Orléans, de par le plus faible taux d'élèves en situation de pauvreté ; cependant, le Massachusetts est parvenu à maintenir un haut niveau de performance au fil du temps, ce qui témoigne de l'impact de ses stratégies d'investissement public. Depuis 2002 environ, le Massachusetts se classe en tête des États américains dans l'évaluation nationale des performances éducatives (National Assessment of Educational Progress) pour les résultats des élèves dans toutes les matières. Et il se situe à des niveaux comparables à ceux des pays les mieux classés dans PISA (Kelly et al., 2013).

Or cela n'est pas dû à un mouvement de privatisation généralisé : le seul organisme habilité à autoriser les charter schools au Massachusetts, le Conseil d'éducation de l'État, limite le développement de ces établissements à $6 \%$ des 
1850 établissements de l'État. Les charter schools virtuelles ne sont pas non plus autorisées, bien qu'une nouvelle option proposant une " éducation collaborative » puisse ouvrir la voie à la mise en œuvre d'une part d'apprentissage virtuel dans deux des écoles existantes. Les nouvelles exigences réglementaires, mises en œuvre en 2014, ont en outre renforcé le système de reddition de comptes des charter schools et ont rendu difficile l'exclusion des élèves.

Mais l'histoire de l'ascension fulgurante de l'État du Massachusetts vers l'excellence s'est écrite bien avant cette récente apparition des charter schools, qui ne sont qu'une réponse aux incitations fédérales à la "course vers l'excellence ». L'histoire débute en 1992, avec une décision de justice dans l'affaire Hancock contre Driscoll, réclamant une refonte du système de financement des écoles dans cet État. La formule de financement de l'école adoptée en 1993 a entraîné des investissements nettement plus importants dans les établissements les plus défavorisés, grâce une formule de calcul pondérée dont l'objectif était d'égaliser le financement de chaque établissement (État plus effort local), en y ajoutant une part additionnelle basée sur la proportion d'élèves à faible revenu et de non anglophones dans un district.

Cette approche progressive a permis de stimuler les investissements mais également les résultats scolaires, car l'État a également entrepris une réforme globale de l'enseignement comprenant de nouveaux standards et de nouvelles évaluations requérant un enseignement et un apprentissage plus ambitieux d'un point de vue intellectuel. Outre le financement accru et plus équitable des établissements, d'autres initiatives ont été prises, comprenant : des standards à l'échelle de l'État pour les élèves, les enseignants, les écoles et les districts; de nouveaux cadres curriculaires afin d'orienter l'enseignement et les évaluations de l'État; un temps d'apprentissage plus important dans les matières de base ; des investissements dans les technologies; des exigences de formation initiale et d'accréditation des enseignants plus strictes ; un meilleur accès à des opportunités de formation continue de qualité pour les enseignants et les chefs d'établissements.

En 1994, l'État du Massachusetts a en outre adopté un plan pour le développement professionnel - le premier de son histoire - qui a conduit à la mise en place de plusieurs initiatives: des formation d'été intensives dans les matières clés, comme les mathématiques et les sciences; des fonds dédiés aux districts pour soutenir le développement professionnel des enseignants; des critères de renouvellement de certification basés sur la formation continue ; et la formulation d'un nouvel ensemble de standards et d'attentes pour les évaluations locales. Le programme "Attracting Excellence to Teaching » [Attirer l'excellence dans l'enseignement] a également été mis en place afin de subventionner la préparation de nouveaux entrants qualifiés dans la profession. Qui plus est, le financement de l'État en faveur des programmes locaux pour la petite enfance a augmenté de $500 \%$ dans les quatre premières années de la réforme, et plus encore dans les années suivantes. 
Le Massachusetts a depuis maintenu, chaque année, son avance sur les autres États, en poursuivant ces programmes d'investissements publics. Des études ont montré que d'autres États ayant réalisé des progrès similaires sur le long terme, comme le Connecticut, le New Jersey et le Vermont, ont suivi une voie comparable (Darling-Hammond, 2010 ; Murnane et Levy, 1996). Ces exemples apportent ainsi la preuve que des investissements en faveur de l'enseignement public, lorsqu'ils sont faits judicieusement et de manière systémique, peuvent contribuer à améliorer la qualité de l'éducation.

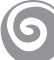

Malgré les efforts considérables consentis dans certaines villes et certains États pour mettre en ouvre des réformes fondées sur le marché et l'émergence d'un grand nombre d'établissements privés grâce au soutien du niveau fédéral, peu de preuves existent d'améliorations systémiques associées à ces initiatives. S'il existe certainement des charter schools très performantes - dont certaines ont acquis cette distinction sans écrémer ni tailler dans leurs populations d'élèves -, des preuves solides indiquent que les réformes fondées sur le marché ont conduit à une stratification et à une ségrégation accrue. Ces réformes poussent en outre les établissements à déployer des efforts considérables afin de sélectionner et de ne garder que les élèves sans difficulté d'apprentissage, en excluant ceux ayant des besoins plus importants.

À l'opposé, des États comme le Massachusetts, qui sont parvenus à réaliser des progrès sûrs et durables en matière de performance et à réduire les écarts de réussite, ont réalisé des investissements publics stratégiques en faveur de l'équité, amélioré la formation initiale et continue des enseignants et renforcé les mesures de soutien aux enfants.

La question fondamentale est de savoir si les États-Unis vont continuer sur la voie de la privatisation, en intensifiant le mouvement d'expansion des charter schools et en développant les programmes de chèques éducation, ou s'ils vont chercher à reconquérir l'éducation publique par des réformes qui la préserveront en tant que bien public.

\section{BIBLIOGRAPHIE}

ADAMSON F. (2010) : How Does Context Matter? Comparing Achievement Scores, Opportunities to Learn, and Teacher Preparation across Socio-Economic Quintiles in TIMSS and PISA (thèse de doctorat non publiée), Stanford University, Stanford, CA.

DARLING-HAMMOND L., AHIGHTOWER A, HUSBANDS J., et al. (2005) : Instructional leadership for systemic change: The story of San Diego's reform (No. 3), Lanham, Maryland : The Rowman \& Littlefield Publishing Group, Inc.

DARLING-HAMMOND L. (2010) : The Flat World and Education: How America's commitment to Equity will Determine our Future, New York, NY : Teachers College Press. 
ELMORE R., BURNEY D. (1997) : Investing in Teacher Learning: Staff Development and Instructional Improvement in Community School District \#2, New York City, New York, NY : National Commission on Teaching \& America's Future.

JACKSON C. K., JOHNSON R., PERSICO C. (2014) : The Effect of School Finance Reforms on the Distribution of Spending Academic Achievement and Adult Outcomes (No. w20118), National Bureau of Economic Research.

KELLY D., NORD C. W., JENKINS F., et al. (2013) : Performance of US 15-Year-Old Students in Mathematics, Science, and Reading Literacy in an International Context. First Look at PISA 2012, NCES 2014-024, Washington, DC: National Center for Education Statistics.

MURNANE R. J., LEVY F.(1996) : Teaching the New Basic Skills. Principles for Educating Children To Thrive in a Changing Economy, New York, NY : Free Press.

NATIONAL CENTER FOR EDUCATION STATISTICS (NCES) (2015) : The Condition of Education: Charter School Enrollment, en ligne : [https://goo.gl/gQLzFP].

WILLIAMS J. (2013) : "Most students leaving RSD's 4 closed, failing schools are headed to other substandard schools", The Lens, 11 juin 2013, en ligne : [https://goo. $\mathrm{gl} / 6 \mathrm{CmUEQ}]$ 
